\title{
Molecularly-defined diffuse leptomeningeal glioneuronal tumor (DLGNT) comprises two subgroups with distinct clinical and genetic features
}

Maximilian Y. Deng ${ }^{1,2}$, Martin Sill ${ }^{1,3}$, Jason Chiang ${ }^{4}$, Jens Schittenhelm ${ }^{5}$, Martin Ebinger ${ }^{6}$, Martin U. Schuhmann 7, Camelia-Maria Monoranu ${ }^{8}$, Till Milde 1, 9, 10, 11, Andrea Wittmann ${ }^{1,2}$, Christian Hartmann ${ }^{12}$, Clemens Sommer ${ }^{13}$, Werner Paulus ${ }^{14}$, Jutta Gärtner ${ }^{15}$, Wolfgang Brück ${ }^{16}$, Thomas Rüdiger ${ }^{17}$, Alfred Leipold $^{18}$, Zane Jaunmuktane ${ }^{19,20}$, Sebastian Brandner ${ }^{19}$ 21, Felice Giangaspero ${ }^{22,} 23$, Paolo Nozza ${ }^{24}$, Jaume Mora ${ }^{25}$, Andres Morales la Madrid ${ }^{25}$, Ofelia Cruz Martinez ${ }^{25}$, Jordan R. Hansford ${ }^{26}$, Torsten Pietsch ${ }^{27}$, Anna Tietze ${ }^{28}$, Pablo Hernáiz-Driever ${ }^{29}$, Iris Stoler ${ }^{30}$, David Capper ${ }^{30}$, Andrey Korshunov ${ }^{31}$, 32, David W. Ellison ${ }^{4}$, Andreas von Deimling ${ }^{31,32}$, Stefan M. Pfister ${ }^{1,3,10}$, Felix Sahm ${ }^{31,32 *}$, David T.W. Jones ${ }^{1,2 *}$

${ }^{1}$ Hopp Children's Cancer Center at the NCT Heidelberg (KiTZ), 69120 Heidelberg, Germany

2 Junior Research Group Pediatric Glioma Research, German Cancer Research Center (DKFZ), 69120 Heidelberg, Germany

3 Division of Pediatric Neurooncology, German Cancer Research Center (DKFZ), 69120 Heidelberg, Germany

${ }^{4}$ Department of Pathology, St. Jude Children's Research Hospital, Memphis, TN, USA

${ }^{5}$ Department of Neuropathology, Institute of Pathology and Neuropathology and Comprehensive Cancer Center Tübingen-Stuttgart, University Hospital of Tübingen, Tübingen, Germany

${ }^{6}$ Department of Pediatric Hematology/Oncology, Children's University Hospital, Tübingen, Germany

7 Department of Neurosurgery, Division of Pediatric Neurosurgery, University Hospital Tübingen, Tübingen, Germany

${ }^{8}$ Institute of Pathology, Department of Neuropathology, University of Würzburg, Comprehensive Cancer Center (CCC) Mainfranken, Würzburg, Germany

${ }^{9}$ Clinical Cooperation Unit Pediatric Oncology, German Cancer Research Center (DKFZ) and German Consortium for Translational Cancer Research (DKTK), 69120 Heidelberg, Germany

${ }^{10}$ Department of Pediatric Oncology, Hematology and Immunology, University Hospital Heidelberg, 69120 Heidelberg, Germany

${ }^{11}$ KiTZ Clinical Trial Unit (ZIPO), 69120 Heidelberg, Germany

${ }^{12}$ Department of Neuropathology, Hannover Medical School, Hannover, Germany

${ }^{13}$ Institute of Neuropathology, University Medical Center of the Johannes Gutenberg-University, Mainz, Germany

${ }^{14}$ Institute of Neuropathology, University Hospital Münster, Münster, Germany

${ }^{15}$ Department of Pediatrics and Adolescent Medicine, Division of Pediatric Neurology, University of Göttingen Medical Center, Göttingen, Germany

${ }^{16}$ Department of Neuropathology, University of Göttingen Medical Center, Göttingen, Germany

${ }^{17}$ Institute of Pathology, Klinikum Karlsruhe, Karlsruhe, Germany

${ }^{18}$ Department of Pediatrics, Klinikum Karlsruhe, Karlsruhe, Germany

19 Division of Neuropathology, National Hospital for Neurology and Neurosurgery, University College London NHS Foundation Trust, London, UK

${ }^{20}$ Department of Molecular Neuroscience, UCL Institute of Neurology, Queen Square, London WC1N 3BG, UK 
${ }^{21}$ Department of Neurodegeneration, UCL Institute of Neurology, Queen Square, London WC1N 3BG, UK

22 IRCCS Neuromed, Pozzilli, Italy

${ }^{23}$ Department of Radiological, Oncological and Anatomo-pathological Science, Sapienza University of Rome, Rome, Italy

${ }^{24}$ Pathology Unit, Istituto Giannina Gaslini, Genova, Italy

25 Department of Pediatric Hemato-Oncology, Hospital Sant Joan de Deu, Esplugues de Llobregat, Barcelona, Spain

${ }^{26}$ Children's Cancer Centre, Royal Children's Hospital, Murdoch Children's Research Institute, Department of Paediatrics, University of Melbourne, Melbourne, Australia

${ }^{27}$ Institute of Neuropathology, Brain Tumor Reference Center of the Society for Neuropathology and Neuroanatomy, University of Bonn Medical Center, Bonn, Germany

${ }^{28}$ Institute of Neuroradiology, Charité Universitätsmedizin, Corporate member of Freie Universität Berlin, Humboldt Universität zu Berlin, and Berlin Institute of Health, Berlin, Germany

29 Department of Pediatric Oncology/Hematology, Charité Universitätsmedizin, Corporate member of Freie Universität Berlin, Humboldt Universität zu Berlin, and Berlin Institute of Health, Berlin, Germany

${ }^{30}$ Department of Neuropathology, Charité Universitätsmedizin, Corporate member of Freie Universität Berlin, Humboldt Universität zu Berlin, and Berlin Institute of Health, Berlin, Germany

${ }^{31}$ Clinical Cooperation Unit Neuropathology, German Cancer Consortium, German Cancer Research Center, 69120 Heidelberg, Germany

32 Department of Neuropathology, Institute of Pathology, University Hospital Heidelberg, 69120 Heidelberg, Germany

Keywords: Diffuse glioneuronal leptomeningeal tumor; pediatric; brain tumor; methylation; prognostic; subgroup; survival; BRAF; MAPK; NTRK; RAF1

\section{Corresponding author:}

David T. W. Jones, PhD

Junior Research Group Pediatric Glioma Research (G702)

German Cancer Research Center (DKFZ)

Im Neuenheimer Feld 280

69120 Heidelberg

Germany

Phone: +49 6221564675

Fax: +49 6221564639

E-Mail: david.jones@kitz-heidelberg.de 


\begin{abstract}
Diffuse leptomeningeal glioneuronal tumors (DLGNT) represent rare CNS neoplasms which have been included in the 2016 update of the WHO classification. The wide spectrum of histopathological and radiological features can make this enigmatic tumor entity difficult to diagnose. In recent years, large-scale genomic and epigenomic analyses have afforded insight into key genetic alterations occurring in multiple types of brain tumors, and provide unbiased, complementary tools to improve diagnostic accuracy. Through genome-wide DNA methylation screening of $>25,000$ tumors, we discovered a molecularly distinct class comprising 30 tumors, mostly diagnosed histologically as DLGNTs. Copy-number profiles derived from the methylation arrays revealed unifying characteristics, including loss of chromosomal arm $1 p$ in all of cases. Furthermore, this molecular DLGNT class can be subdivided into two subgroups (DLGNT-MC-1 and DLGNT-MC-2), with all DLGNT-MC-2 additionally displaying a gain of chromosomal arm 1q. Codeletion of 1p/19q, commonly seen in IDH-mutant oligodendroglioma, was frequently observed in DLGNT, especially in DLGNT-MC-1 cases. Both subgroups also had recurrent genetic alterations leading to an aberrant MAPK/ERK pathway, with KIAA1549:BRAF fusion being the most frequent event. Other alterations included fusions of NTRK1/2/3 and TRIM33:RAF1, adding up to a MAPK/ERK pathway activation identified in $80 \%$ of cases. In the DLGNT-MC-1 group, age at diagnosis was significantly lower (median 5 vs 14 years, $p<0.01$ ) and clinical course less aggressive $(5$-yr OS $100 \%$, vs $43 \%$ in DLGNT-MC-2). Our study proposes an additional molecular layer to the current histopathological classification of DLGNT, of particular use for cases without typical morphological or radiological characteristics, such as diffuse growth and radiologic leptomeningeal dissemination. Recurrent $1 \mathrm{p}$ deletion and MAPK/ERK pathway activation represent diagnostic biomarkers and therapeutic targets, respectively - laying the foundation for future clinical trials with e.g. MEK inhibitors that may improve the clinical outcome of patients with DLGNT.
\end{abstract}




\section{Introduction}

Diffuse leptomeningeal glioneuronal tumors (DLGNTs) rare CNS neoplasms preferentially occur in children but several cases in adult patients have also been reported [9, 10,31]. A gender predilection is apparent, with males being predominantly affected. Among 81 patients in 20 different reports, $62 \%(50 / 81)$ were male and $38 \%(31 / 81)$ female $[1,3,6,8-10,12,13,15,16,19,25,27,29-31,33,35-37]$. A summary of characteristics in previous series is given in Table 1. Although most tumors display a slow progression, aggressive cases were occasionally encountered $[12,29,31]$. The largest series with follow-up data, of 24 patients, showed 9 deaths within 3 to 21 years [31]. Treatment regimens, where given, typically included surgical resection, radiotherapy and/or chemotherapy.

In previous reports, the majority of DLGNTs was radiologically characterized by leptomeningeal enhancement on MRI in the posterior fossa, around the brain stem and the spinal cord. Small cystic or nodular T2 - hyperintense lesions were frequently encountered along the subpial surface of the brain and spinal cord. Discrete intraparenchymal lesions were also found, especially in the spinal cord [1, 12, 15, 29, 31, 35]. However, in a recent study by Chiang et al. comprising 5 patients, leptomeningeal dissemination was not seen radiologically in any of the cases, suggesting that DLGNTs do not necessarily present with gross leptomeningeal dissemination on MRI [9].

Histologically, DLGNTs have been described as low- to moderate-cellularity lesions consisting of relatively monomorphous oligodendrocyte-like cells with round to oval nuclei, inconspicuous nucleoli and a 'glioneural commitment' [15], embedded in a desmoplastic or myxoid leptomeningeal stroma [29, 31]. While a low mitotic activity was most commonly observed, features of anaplasia were also seen in a number of cases [31, 36]. Immunohistochemical features of the tumor cells appear to include strong reactivity for OLIG2, MAP2 and S100 with variable expression for GFAP and synaptophysin. Immunostaining for NeuN, EMA and mutant IDH1 (R132H) were negative $[9,29,31,35]$. Although typically displaying a low Ki-67 proliferation index, several cases with elevated proliferation were reported [29,31,35], with less favorable outcome associated with an index $>4 \%[31]$. The wide spectrum of histological and radiological features encountered, as well as its rarity and relatively recent inclusion in the WHO classification, can make this tumor entity difficult to diagnose.

In recent years, large-scale genomic and epigenomic analyses have provided insight into key genetic alterations occurring in multiple types of brain tumors. For example, various mechanisms converging on an aberrant activation of the mitogen-activated protein kinase/extracellular signal-regulated kinase (MAPK/ERK) signaling pathway [20, 21, 39,43 ] represent a hallmark of pediatric low-grade gliomas, with KIAA1549:BRAF fusion being the most frequent event [23, 24, 28]. Alternative mechanisms such as NTRK and RAF1 rearrangements, also converging on activation of the MAPK/ERK pathway, were also discovered $[2,23,24,43]$.

The existence of KIAA1549:BRAF fusions in DLGNT has also been described in recent publications, in conjunction with deletions of chromosomal arm $1 p$ or 1p/19q co-deletions but in the absence of IDH mutation (Table 1) [1, 9, 12, 30, $32,33,35]$.

Herein, we performed a DNA methylation, copy number and targeted sequencing-based molecular characterization of this entity to clarify the underlying molecular and biological mechanisms, and to detect potentially targetable alterations. In addition, molecular features were correlated with clinical, neuropathological and radiological findings, leading to the delineation of two molecularly and clinically distinct subsets of DLGNT. 


\section{Experimental procedures}

\section{Tumor Material and Patient Population}

Tumor samples and clinical data were provided by multiple international collaborating centers and collected at the German Cancer Research Center (DKFZ, Heidelberg, Germany) with approval from the respective institutional review boards. Informed consent was obtained from all patients according to local protocols. Clinical patient details are listed in Table $\mathrm{X}$.

Through genome-wide DNA methylation screening of $>25,000$ tumors, we discovered a distinct class comprising 30 tumors, with the most common histological diagnosis being DLGNT. All patients within this distinct class were included for further investigation.

\section{DNA Methylation Profiling}

The Illumina Infinium HumanMethylation450 (450k) array and Illumina Infinium MethylationEPIC (EPIC) array were used to obtain genome-wide DNA methylation profiles, according to the manufacturer's instructions (Illumina, San Diego, USA). Data was generated at the Genomics and Proteomics Core Facility of the DKFZ (Heidelberg, Germany) and St. Jude Children's Research Hospital (Memphis, USA). DNA methylation data was generated from both freshfrozen and formalin-fixed paraffin-embedded (FFPE) tissue samples. On-chip quality metrics of all samples were carefully controlled.

Copy-number variation (CNV) analysis from 450k and EPIC methylation array data was performed using the conumee Bioconductor package version 1.12.0.

All computational analyses were performed in $\mathrm{R}$ version 3.4.1 ( $\mathrm{R}$ Development Core Team, 2018). Raw signal intensities were obtained from IDAT-files using the minfi Bioconductor package version 1.24.0 [4]. IIlumina EPIC and 450k samples were merged to a combined data set by selecting the intersection of probes present on both arrays (combineArrays function, minfi). Each sample was individually normalized by performing a background correction (shifting of the $5 \%$ percentile of negative control probe intensities to 0 ) and a dye-bias correction (scaling of the mean of normalization control probe intensities to 10,000) for both color channels. Subsequently, a correction for the type of material tissue (FFPE/frozen) and array (450k/EPIC) was performed by fitting univariate, linear models to the log2-transformed intensity values (removeBatchEffect function, limma package version 3.34.5). The methylated and unmethylated signals were corrected individually. Beta-values were calculated from the retransformed intensities using an offset of 100 (as recommended by Illumina).

Before further analysis the following filtering criteria were applied: Removal of probes targeting the $X$ and $Y$ chromosomes $(n=11,551)$, removal of probes containing a single-nucleotide polymorphism (dbSNP132 Common) within five base pairs of and including the targeted CpG-site $(n=7,998)$, probes not mapping uniquely to the human reference genome (hg19) allowing for one mismatch $(n=3,965)$, and 450k array probes not included on the EPIC array. In total, 428,230 probes were kept for downstream analysis.

To perform unsupervised dimension reduction, the remaining probes were used to calculate the 1-variance weighted Pearson correlation between samples. The resulting distance matrix was used as input for t-SNE analysis (tDistributed Stochastic Neighbor Embedding; Rtsne package version 0.13) [41]. The following non-default parameters were applied: theta=0, pca=F, max_iter $=2500$ perplexity $=20$.

To perform unsupervised hierarchical clustering, the 10000 probes with highest standard deviation were selected to calculate the Euclidean distance between samples, followed by applying Wards linkage method for sample clustering. In the heatmap representation probes were reordered by complete linkage hierarchical clustering of the Euclidean distance between probes.

To evaluate focal amplifications and deletions and chromosomal gains and losses, we visually inspected copy-number profiles of each case. Candidate genes and their $3^{\prime}$ and 5' intergenic neighborhood were further investigated using the Integrative Genomic Viewer (IGV) [40] for the presence of breakpoints, as an indication for potential gene fusions. 


\section{Histopathology}

Where possible, hematoxylin and eosin (H\&E) stained formalin fixed paraffin embedded (FFPE) sections from tumors in the DLGNT methylation class $(n=14 / 30)$ were reviewed by an experienced neuropathologist (FS or DWE) according to WHO 2016 guidelines.

\section{Gene Panel Sequencing/ Single Gene Testing}

For the detection of single nucleotide variations, small insertions and deletions (indels), fusions, and copy number aberrations, a subset of samples $(n=5)$ was subjected to customized enrichment/hybrid-capture-based next generation sequencing gene panel analysis covering 130 genes of particular relevance in brain tumors, as previously described [34]. The presence of KIAA1549-BRAF fusion transcripts was tested in four samples by reverse transcription polymerase chain reaction (RT-PCR). Additionally, single gene testing was performed via immunostaining for BRAF p.V600E, histone H3 p.K27M and IDH1 p.R132H mutant proteins in five cases.

\section{Statistics}

Overall survival (OS) and progression-free survival (PFS) was evaluated by Kaplan-Meier analysis and log-rank test. Binary and categorical patient characteristics between subgroups were compared via two-sided Fisher's exact test. P-values $<0.05$ were considered significant. 


\section{Results}

\section{DNA methylation profiling defines molecular DLGNT classes}

Recent large-scale genomic and epigenomic studies demonstrated that the DNA methylation pattern of tumor cells can provide valuable information for tumor classification. These DNA methylation patterns are thought to represent a preserved 'molecular memory' of the respective cell of origin throughout the course of disease, thus displaying a distinct signature for each biologically discrete tumor entity [7, 17, 18, 38].

Through genome-wide DNA methylation screening of $>25,000$ tumors in the extended Heidelberg cohort, an apparently distinct class comprising 30 tumors was detected, of which most were diagnosed histologically as DLGNT. A subsequent focused unsupervised analysis of DNA methylation patterns of these tumors together with 129 wellcharacterized reference samples representing CNS tumors of known histological and/or molecular subtype confirmed the distinct nature of this class (Figure 1).

Furthermore, this molecular DLGNT group could be divided into two subclasses (Figure 1b) through DNA methylation clustering, thus we subsequently refer to these groups as DLGNT-methylation class (MC)-1 ( $n=17)$ and DLGNT-MC-2 $(n=13)$.

\section{Initial diagnoses of DLGNT}

Initial diagnoses were available for 15/17 DLGNT-MC-1 and 13/13 DLGNT-MC-2 tumors. 'Diffuse leptomeningeal glioneuronal tumor' represented the most frequent initial diagnosis in both subgroups (DLGNT-MC-1: 8/15, 53\%; DLGNT-MC-2: 5/13, 38\%). Various other tumors were diagnosed as primitive neuroectodermal tumors WHO IV (DLGNT-MC-1: 2/15, 13\%; DLGNT-MC-2: 2/13, 15\%), pilocytic astrocytoma WHO I (2/15, 13\% in DLGNT-MC-1; 2/13, $15 \%$ in DLGNT-MC-2) or anaplastic astrocytoma WHO III (1/15, 7\% in DLGNT-MC-1; 2/13, 15\% in DLGNT-MC-2) as well as single cases as extraventricular neurocytoma WHO grade II, oligodendroglioma WHO III and ganglioglioma WHO grade I. Thus, the biologically-defined DLGNT class appears to be associated with a substantial morphological heterogeneity, leading to a substantial risk of misdiagnosis when relying on histology alone. In order to examine this further, as many cases as possible were re-evaluated by experienced neuropathologists (FS, DWE).

HE-stained FFPE sections were available for 9 DLGNT-MC-1 and 5 DLGNT-MC-2 tumors. Histologically, they presented as moderate- to high-cellularity neoplasms with low mitotic activity. Tumor cells most commonly displayed isomorphic, round to oval nuclei $(11 / 14,79 \%)$ with inconspicuous nucleoli, with some other tumors (4/14, $29 \%)$ displaying small eccentric nuclei.

Oligodendroglioma-like perinuclear haloes were more frequently observed in the DLGNT-MC-1 subgroup (8/9, 89\% vs $2 / 5,40 \%$ in DLGNT-MC-2). The tumors displayed desmoplastic and myxoid areas, sometimes with fibrillary components. In both subgroups, microcysts and neuropil-like islands were observed. In addition, DLGNT-MC-1 and DLGNT-MC-2 occasionally harbored eosinophilic granular bodies and Rosenthal fibers together with vascular proliferation. Hyalinization of the vascular walls was encountered in the periphery of the biopsies in two DLGNT-MC1 cases. Microscopic leptomeningeal involvement was detected in 6 cases, in which 5 cases were inconspicuous at radiological presentation. Presence of gemistocytes was demonstrated in one DLGNT-MC-2 biopsy. Signs of necrosis were absent in all tumor specimen.

\section{Varying radiological features of DLGNTs}

Comprehensive radiological data were available for 10 DLGNT-MC-1 and 5 DLGNT-MC-2 patients. Evaluation of available MRI series revealed T2 hyperintense cystic lesions or nodular masses both along the surface and also within the parenchyma of the spinal cord (14/15, 93\%) with occasional involvement of the cerebellum (5/15, 33\%). The overall proportion of cases reported as spinal, however, was lower (11/13, 85\% in DLGNT-MC-1, 7/12, 58\% in DLGNTMC-2) than that for those cases with MRI data available. Hereby, we excluded 3 cases due to their preselection based on their exclusive spinal involvement, previously reported in Chiang et al. [9]. During the process of tumor progression, supratentorial regions including the cerebral cortex, basal ganglia and thalamus were additionally 
affected in a subset cases. Only one DLGNT-MC-1 patient displayed intraparenchymal lesions exclusively in the left frontal lobe of the cerebral cortex without spinal involvement. Some of the cystic or nodular lesions showed contrast agent uptake, while others did not, suggesting a generally mixed presentation with variable enhancement.

Previous studies reported that one of the key features of DLGNTs is its diffuse leptomeningeal enhancement on MRI. In our series, contrast enhancement of the leptomeninges was more frequently observed in DLGNT-MC-2 (4/5 vs $3 / 10$ in DLGNT-MC-1; $p=0.12$ ), where it particularly involved the surface of the cerebellum, basal cisterns and the spinal cord. The surface of these regions appeared to be covered by a thin 'sugar coat', in some cases extending from the brain stem down to the conus medullaris, and spanning multiple segments. In a few cases, spinal nerve roots appeared to be thickened. Leptomeningeal dissemination throughout the central nervous system was observed in several patients with disease progression.

Importantly, however, a considerable subset of DLGNTs as defined here did not present with leptomeningeal enhancement or dissemination radiologically (7/10 in DLGNT-MC-1, 1/5 in DLGNT-MC-2), supporting the notion that leptomeningeal enhancement on MRI should not be viewed as an essential criterion for diagnosing DLGNT [9].

\section{DLGNT-MC-1 and DLGNT-MC-2 show distinct copy-number profiles}

Copy-number profiles derived from DNA methylation arrays revealed some unifying characteristics of biologicallydefined DLGNT, including loss of chromosomal arm 1p in 100\% of cases. All DLGNT-MC-2, but only 6/17 (35\%) of DLGNT-MC-1 showed an additional gain of chromosomal arm 1q $(p<0.001)$. Co-deletion of $1 p / 19 q$ was frequently encountered in the DLGNT-MC-1 group ( $8 / 17,47 \%$ vs $2 / 13,15 \%$ in DLGNT-MC-2; $p=0.12)$. In contrast, gain of chromosome 8 was more commonly observed in DLGNT-MC-2 (7/13, 54\% vs $1 / 17,6 \%$ in DLGNT-MC-1; $p<0.01)$. Homozygous deletion of CDKN2A/B was found in one DLGNT-MC-2 with KIAA1549:BRAF fusion.

\section{Genetic Rearrangements Leading to Aberrant MAPK/ERK Pathway}

Fusion of KIAA1549:BRAF represented the most recurrent genetic alteration after $1 p$ loss in both subgroups (11/17, $65 \%$ in DLGNT-MC-1 vs $9 / 13,69 \%$ in DLGNT-MC-2). This alteration, leading to constitutive activation of the MAPK/ERK pathway, is a well-known driving oncogenic event in other (particularly pediatric) low-grade gliomas [20-23].

Multiple additional fusions resulting in an aberrant MAPK/ERK pathway were indicated by copy number alterations in DLGNT-MC-1 cases, including fusions involving NTRK1/2/3 present in one case each with currently undetermined fusion partners. In one DLGNT-MC-2 case, for which RNA sequencing data were available, a TRIM33:RAF1 fusion was discovered. RAF1 is a human homolog of the v-raf gene from a transforming retrovirus and involved in cell proliferation, survival, migration and a range of other cellular processes [5, 42]. RAF1 fusions have been reported to possess constitutive kinase activity and transforming ability, underlining their role in tumorigenesis, especially in pilocytic astrocytoma $[11,14,24]$. All MAPK pathway alterations mentioned above were mutually exclusive.

\section{Targeted Sequencing/Next-Generation (NGS) Gene Panel Sequencing}

By using next-generation (NGS) gene panel sequencing and reverse transcription polymerase chain reaction (RT-PCR), we additionally analyzed ten samples, including seven DLGNT-MC-1 and three DLGNT-MC-2 cases. The presence of a KIAA1549-BRAF fusion had already been detected in the copy-number profiles of these samples. Immunostainings for BRAF p.V600E, histone H3 p.K27M and IDH1 p.R132H mutant proteins were available for additionally one DLGNTMC-1 and one DLGNT-MC-2 cases. Evaluable data in seven cases confirmed KIAA1549-BRAF fusions with KIAA1549 exon 15 fusing to BRAF exon 11 in two DLGNT-MC-1 cases and to BRAF exon 9 in three DLGNT-MC-1 and one DLGNTMC-2 cases. Fusion of KIAA1549 exon 13 to BRAF exon 11 was demonstrated in one DLGNT-MC-2 sample.

None of the cases displayed alterations in IDH1 R132 (0/10), IDH2 R172 (0/5), BRAF V600E (0/7), H3F3A G34 (0/5) and H3F3A K27 (0/8). Point mutation in the promoter region of TERT (C228T and C228A) were encountered in 2/6 DLGNT-MC-2 patients, but not in any of the 9 DLGNT-MC-1 cases tested. One tumor sample exhibited a point mutation in ATRX and a frameshift insertion in BCOR; however the functional relevance of these alterations in this context remains unknown.

\section{Clinical Characteristics and Outcome}


We correlated the genetic and methylation data of both subgroups with available basic clinical parameters. Age at diagnosis was significantly lower in DLGNT-MC-1 patients (median: 5 vs 14 years in DLGNT-MC-2; p<0.01). Only one patient whose tumor was classified as 'DLGNT-MC-1' was older than 18, whereas DLGNT-MC-2 showed a clear predilection for older age at diagnosis (range: 5-47 vs 2-23 years in DLGNT-MC-1).

Both methylation subgroups displayed a male predominance (M:F 1.4:1 in DLGNT-MC-1, 1.6:1 in DLGNT-MC-2).

DLGNTs were located throughout the central nervous system, but preferentially in the spinal cord in both subgroups $(11 / 13,85 \%$ in DLGNT-MC-1 and 7/12, 58\% in DLGNT-MC-2), often spanning across multiple segments. Most patients exhibited multiple tumor sites at presentation (Figure 5), making it challenging to assess the most common site of the primary lesion. No association between tumor location and a specific molecular alteration was identified.

In DLGNT-MC-1 patients with available follow-up data $(n=9)$, disease progression was observed in 2 cases (2/9). All DLGNT-MC-1 patients were alive at the time of latest follow-up. Outcome data of DLGNT-MC-2 patients ( $n=8$ ) presented a significantly more aggressive clinical course with $7 / 8$ patients displaying disease progression. In addition, four DLGNT-MC-2 patients (4/8) eventually died after 10, 50, 52 and 173 months from initial diagnosis. Two further DLGNT-MC-2 patients were transferred into palliative care after 17 and 132 months due to dismal physical condition, and were thereafter lost to follow-up. Although based on relatively small numbers, we believe that DLGNT-MC-2 tumors present with a significantly more aggressive clinical course (5-year OS $43 \%$ vs $100 \%$ in DLGNT-MC-1, p<0.05) (Figure 6).

Due to the variable initial histological and imaging features of the tumors of these two distinct methylation classes, a wide spectrum of treatment regimens was applied, including surgical resection, chemotherapy and radiotherapy. Thus, no conclusions can currently be drawn as to the efficacy of particular therapeutic regimes. 


\section{Discussion}

In the past, various terms were attributed to DLGNTs, such as 'disseminated oligodendroglial-like leptomeningeal tumor of childhood' [31], 'superficially disseminated glioma in children' [1], 'primary disseminated leptomeningeal oligodendroglioma' [6] or 'diffuse leptomeningeal neuroepithelial tumor' [35], emphasizing the leptomeningeal involvement. However, a recent report comprising five cases by Chiang et al. characterized DLGNTs as low-grade spinal glioneuronal tumors without conspicuous radiological features at presentation. Leptomeningeal dissemination on MRI was not visible in a considerable proportion of molecularly-defined DLGNTs in our series, thus should not be applied as mandatory criterion for diagnosis. A characteristic appearance of multicystic or nodular, T2 hyperintense lesions in the cerebellum and spinal cord (as part of the multi-focal presentation) was observed in all 30 cases.

Microscopic leptomeningeal lesions were detected during neuropathological review in 6/14 (43\%) tumors, whereby MRI was inconspicuous in 5 cases. However, morphological classification of DLGNT appeared to be difficult based on histopathology and imaging only. Although DLGNT represented the most common initial diagnosis in this molecularlydefined series, a considerable subset of tumors were initially designated to other tumor entities including primitive neuroectodermal tumors WHO grade IV, pilocytic astrocytoma WHO grade I, anaplastic astrocytoma WHO grade III, anaplastic oligodendrogioma WHO grade III, extraventricular neurocytoma WHO grade II or ganglioglioma WHO grade I. In particular, the high prevalence of morphological 'oligodendroglial' elements in DLGNT may suggest that some tumors currently diagnosed as 'pediatric-type' oligodendroglioma could belong to the DLGNT molecular group.

A number of the characteristic features of DLGNT as described here, however, are not represented in the differential diagnostic entities mentioned above. Co-deletions of $1 p / 19 q$ are absent in pilocytic astrocytoma but are observed in adult oligodendroglioma. In contrast to adult oligodendroglioma, however, IDH mutations were absent in DLGNTs, while the KIAA1549:BRAF fusion, usually associated with pilocytic astrocytoma, was common. The cell of origin for this molecular tumor class remains unclear, but the mixture of partially oligodendroglial-like and partially PA-like molecular features could tentatively suggest a precursor population just upstream of this lineage segregation.

Our study identified two molecular DLGNT subgroups (DLGNT-MC-1 and DLGNT-MC-2) with distinct molecular and clinical features. Characteristics common to both classes of DLGNT include loss of chromosomal arm 1p. This is variably associated with 19q co-deletion, in keeping with previous reports $[1,9,12,30,32,33,35]$. Furthermore, all tumors in the DLGNT-MC-2 subgroup additionally display gain of chromosomal arm 1q, which was not reported to date.

Recurrent genetic alterations converging on an aberrant activation of the MAPK/ERK pathway were also highlighted in our series, with KIAA1549:BRAF fusion being the most frequent event (20/30 cases, 66\%), in keeping with previous studies [9, 12, 32]. Other alterations including fusions of NTRK1/2/3 and TRIM33:RAF1 were also detected. Both, NTRK and RAF1 rearrangements result in a constitutive activation of the MAPK/ERK pathway and are known to facilitate tumorigenesis $[2,23,24,43]$. Thus, we conclude that an aberrant activation of the MAPK/ERK pathway together with loss of $1 \mathrm{p}$ are hallmark alterations of the molecularly-defined DLGNT entity.

Notably, age at diagnosis was significantly lower (median: 5 vs 14 years, $p<0.01$ ) and clinical outcome less aggressive in DLGNT-MC-1 patients (5-yr OS $100 \%$ vs $43 \%$ in DLGNT-MC-2, p<0.05). Based on our survival analysis, we would tentatively suggest that DLGNT-MC-1 tumors may best correspond to WHO grade I and DLGNT-MC-2 to WHO II-III.

In summary, our study provides an additional layer to the current histopathological classification of DLGNT, which will be particularly important for the not insignificant fraction of patients where the histological and neuroimaging is not typical of the current description of this entity. Recurrent $1 p$ deletion and MAPK/ERK pathway activation represent diagnostic and therapeutic biomarkers, respectively, and may become important for the stratification into future clinical trials with e.g. MEK or NTRK inhibitors, with the hope of ultimately improving the clinical outcome of patients with DLGNT. 


\section{Acknowledgements}

This work was supported by the Everest Centre for Low-grade Pediatric Brain Tumors (The Brain Tumor Charity, UK), A Kids' Brain Tumor Cure (PLGA) Foundation, the German Cancer Consortium (DKTK), and fellowships from the Mildred-Scheel doctoral program of the German Cancer Aid and the German National Academic Foundation (to M.Y.D.). Sebastian Brandner was partially supported by the National Institute of Health Research (NIHR) UCLH/UCL Biomedical Research Centre. For excellent technical support and expertise we sincerely thank the Microarray Unit of the German Cancer Research Center (DKFZ) Genomics and Proteomics Core Facility, and Hai-Yen Nguyen \& Laura Doerner (Department of Neuropathology, Institute of Pathology, University Hospital Heidelberg). 


\section{Literature}

1 Agamanolis DP, Katsetos CD, Klonk CJ, Bartkowski HM, Ganapathy S, Staugaitis SM, Kuerbitz SJ, Patton DF, Talaizadeh A, Cohen BH (2012) An unusual form of superficially disseminated glioma in children: report of 3 cases. J Child Neurol 27: 727-733 Doi 10.1177/0883073811426500

2 Amatu A, Sartore-Bianchi A, Siena S (2016) NTRK gene fusions as novel targets of cancer therapy across multiple tumour types. ESMO Open 1: e000023 Doi 10.1136/esmoopen-2015-000023

3 Armao DM, Stone J, Castillo M, Mitchell KM, Bouldin TW, Suzuki K (2000) Diffuse leptomeningeal oligodendrogliomatosis: radiologic/pathologic correlation. AJNR Am J Neuroradiol 21: 1122-1126

4 Aryee MJ, Jaffe AE, Corrada-Bravo H, Ladd-Acosta C, Feinberg AP, Hansen KD, Irizarry RA (2014) Minfi: a flexible and comprehensive Bioconductor package for the analysis of Infinium DNA methylation microarrays. Bioinformatics 30: 1363-1369 Doi 10.1093/bioinformatics/btu049

5 Bonner T, O'Brien SJ, Nash WG, Rapp UR, Morton CC, Leder P (1984) The human homologs of the raf (mil) oncogene are located on human chromosomes 3 and 4. Science 223: 71-74

6 Bourne TD, Mandell JW, Matsumoto JA, Jane JA, Jr., Lopes MB (2006) Primary disseminated leptomeningeal oligodendroglioma with 1p deletion. Case report. J Neurosurg 105: 465-469 Doi 10.3171/ped.2006.105.6.465

7 Capper D, Jones DTW, Sill M, Hovestadt V, Schrimpf D, Sturm D, et al (2018) DNA-methylationbased classification of central nervous system tumours. Nature in press:

8 Chen R, Macdonald DR, Ramsay DA (1995) Primary diffuse leptomeningeal oligodendroglioma. Case report. J Neurosurg 83: 724-728 Doi 10.3171/jns.1995.83.4.0724

9 Chiang JCH, Harreld JH, Orr BA, Sharma S, Ismail A, Segura AD, Ellison DW (2017) Low-grade spinal glioneuronal tumors with BRAF gene fusion and $1 \mathrm{p}$ deletion but without leptomeningeal dissemination. Acta Neuropathol 134: 159-162 Doi 10.1007/s00401-017-1728-4

Cho HJ, Myung JK, Kim H, Park CK, Kim SK, Chung CK, Choi SH, Park SH (2015) Primary diffuse leptomeningeal glioneuronal tumors. Brain Tumor Pathol 32: 49-55 Doi 10.1007/s10014-0140187-z

11 Cin H, Meyer C, Herr R, Janzarik WG, Lambert S, Jones DT, Jacob K, Benner A, Witt H, Remke Met al (2011) Oncogenic FAM131B-BRAF fusion resulting from 7q34 deletion comprises an alternative mechanism of MAPK pathway activation in pilocytic astrocytoma. Acta Neuropathol 121: 763-774 Doi 10.1007/s00401-011-0817-z

12 Dodgshun AJ, SantaCruz N, Hwang J, Ramkissoon SH, Malkin H, Bergthold G, Manley P, Chi S, MacGregor D, Goumnerova Let al (2016) Disseminated glioneuronal tumors occurring in childhood: treatment outcomes and BRAF alterations including V600E mutation. J Neurooncol 128: 293-302 Doi 10.1007/s11060-016-2109-x

13 Dyson K, Rivera-Zengotita M, Kresak J, Weaver K, Stover B, Fort J, Rahman M, Pincus DW, Sayour EJ (2016) FGFR1 N546K and H3F3A K27M mutations in a diffuse leptomeningeal tumour with glial and neuronal markers. Histopathology 69: 704-707 Doi 10.1111/his.12983

14 Forshew T, Tatevossian RG, Lawson AR, Ma J, Neale G, Ogunkolade BW, Jones TA, Aarum J, Dalton J, Bailey Set al (2009) Activation of the ERK/MAPK pathway: a signature genetic defect in posterior fossa pilocytic astrocytomas. J Pathol 218: 172-181 Doi 10.1002/path.2558

Gardiman MP, Fassan M, Orvieto E, D'Avella D, Denaro L, Calderone M, Severino M, Scarsello G, Viscardi E, Perilongo G (2010) Diffuse leptomeningeal glioneuronal tumors: a new entity? Brain Pathol 20: 361-366 Doi 10.1111/j.1750-3639.2009.00285.x

16 Gilmer-Hill HS, Ellis WG, Imbesi SG, Boggan JE (2000) Spinal oligodendroglioma with gliomatosis in a child. Case report. J Neurosurg 92: 109-113 

MD, Uzunangelov Vet al (2014) Multiplatform analysis of 12 cancer types reveals molecular classification within and across tissues of origin. Cell 158: 929-944 Doi 10.1016/j.cell.2014.06.049 Hovestadt V, Jones DT, Picelli S, Wang W, Kool M, Northcott PA, Sultan M, Stachurski K, Ryzhova $\mathrm{M}$, Warnatz HJet al (2014) Decoding the regulatory landscape of medulloblastoma using DNA methylation sequencing. Nature 510: 537-541 Doi 10.1038/nature13268 unusual cystic appearance of disseminated low-grade gliomas. Neuroradiology 43: 868-874 Jeuken JW, Wesseling P (2010) MAPK pathway activation through BRAF gene fusion in pilocytic astrocytomas; a novel oncogenic fusion gene with diagnostic, prognostic, and therapeutic potential. J Pathol 222: 324-328 Doi 10.1002/path.2780 Jones DT, Gronych J, Lichter P, Witt O, Pfister SM (2012) MAPK pathway activation in pilocytic astrocytoma. Cell Mol Life Sci 69: 1799-1811 Doi 10.1007/s00018-011-0898-9

Jones DT, Hutter B, Jager N, Korshunov A, Kool M, Warnatz HJ, Zichner T, Lambert SR, Ryzhova M, Quang DAet al (2013) Recurrent somatic alterations of FGFR1 and NTRK2 in pilocytic astrocytoma. Nat Genet 45: 927-932 Doi 10.1038/ng.2682

Jones DT, Kocialkowski S, Liu L, Pearson DM, Backlund LM, Ichimura K, Collins VP (2008) Tandem duplication producing a novel oncogenic BRAF fusion gene defines the majority of pilocytic astrocytomas. Cancer Res 68: 8673-8677 Doi 10.1158/0008-5472.CAN-08-2097

Jones DT, Kocialkowski S, Liu L, Pearson DM, Ichimura K, Collins VP (2009) Oncogenic RAF1 rearrangement and a novel BRAF mutation as alternatives to KIAA1549:BRAF fusion in activating the MAPK pathway in pilocytic astrocytoma. Oncogene 28: 2119-2123 Doi 10.1038/onc.2009.73

Kessler BA, Bookhout C, Jaikumar S, Hipps J, Lee YZ (2015) Disseminated oligodendroglial-like leptomeningeal tumor with anaplastic progression and presumed extraneural disease: case report. Clin Imaging 39: 300-304 Doi 10.1016/j.clinimag.2014.11.018

Louis DN, Ohgaki H, Wiestler OD, Cavenee WK, Ellison DW, Figarella-Branger D, Perry A, Reifenberger G, Von Deimling A (2016) WHO Classification of Tumours of the Central Nervous System. International Agency for Research on Cancer, City

27 Perilongo G, Gardiman M, Bisaglia L, Rigobello L, Calderone M, Battistella A, Burnelli R, Giangaspero $F(2002)$ Spinal low-grade neoplasms with extensive leptomeningeal dissemination in children. Childs Nerv Syst 18: 505-512 Doi 10.1007/s00381-002-0626-8

Pfister S, Janzarik WG, Remke M, Ernst A, Werft W, Becker N, Toedt G, Wittmann A, Kratz C, Olbrich Het al (2008) BRAF gene duplication constitutes a mechanism of MAPK pathway activation in lowgrade astrocytomas. J Clin Invest 118: 1739-1749 Doi 10.1172/JCl33656

Preuss M, Christiansen H, Merkenschlager A, Hirsch FW, Kiess W, Muller W, Kastner S, Henssler A, Pekrun A, Hauch Het al (2015) Disseminated oligodendroglial-like leptomeningeal tumors: preliminary diagnostic and therapeutic results for a novel tumor entity [corrected]. J Neurooncol 124: 65-74 Doi 10.1007/s11060-015-1735-z

30 Rhiew RB, Manjila S, Lozen A, Guthikonda M, Sood S, Kupsky WJ (2010) Leptomeningeal dissemination of a pediatric neoplasm with $1 \mathrm{p} 19 \mathrm{q}$ deletion showing mixed immunohistochemical features of an oligodendroglioma and neurocytoma. Acta Neurochir (Wien) 152: 1425-1429 Doi 10.1007/s00701-010-0674-x

31 Rodriguez FJ, Perry A, Rosenblum MK, Krawitz S, Cohen KJ, Lin D, Mosier S, Lin MT, Eberhart CG, Burger PC (2012) Disseminated oligodendroglial-like leptomeningeal tumor of childhood: a distinctive clinicopathologic entity. Acta Neuropathol 124: 627-641 Doi 10.1007/s00401-0121037-x

32 Rodriguez FJ, Schniederjan MJ, Nicolaides T, Tihan T, Burger PC, Perry A (2015) High rate of concurrent BRAF-KIAA1549 gene fusion and $1 p$ deletion in disseminated oligodendroglioma-like 
leptomeningeal neoplasms (DOLN). Acta Neuropathol 129: 609-610 Doi 10.1007/s00401-015$1400-9$

33 Rossi S, Rodriguez FJ, Mota RA, Dei Tos AP, Di Paola F, Bendini M, Agostini S, Longatti P, Jenkins RB, Giannini C (2009) Primary leptomeningeal oligodendroglioma with documented progression to anaplasia and t(1;19)(q10;p10) in a child. Acta Neuropathol 118: 575-577 Doi 10.1007/s00401009-0565-5

34 Sahm F, Schrimpf D, Jones DT, Meyer J, Kratz A, Reuss D, Capper D, Koelsche C, Korshunov A, Wiestler Bet al (2016) Next-generation sequencing in routine brain tumor diagnostics enables an integrated diagnosis and identifies actionable targets. Acta Neuropathol 131: 903-910 Doi 10.1007/s00401-015-1519-8

35 Schniederjan MJ, Alghamdi S, Castellano-Sanchez A, Mazewski C, Brahma B, Brat DJ, Brathwaite CD, Janss AJ (2013) Diffuse leptomeningeal neuroepithelial tumor: 9 pediatric cases with chromosome 1p/19q deletion status and IDH1 (R132H) immunohistochemistry. Am J Surg Pathol 37: 763-771 Doi 10.1097/PAS.0b013e31827bf4cc

36 Schwetye KE, Kansagra AP, McEachern J, Schmidt RE, Gauvain K, Dahiya S (2017) Unusual highgrade features in pediatric diffuse leptomeningeal glioneuronal tumor: comparison with a typical low-grade example. Hum Pathol: Doi 10.1016/j.humpath.2017.06.004

37 Stodberg T, Deniz Y, Esteitie N, Jacobsson B, Mousavi-Jazi M, Dahl H, Zweygberg Wirgart B, Grillner $L$, Linde $A$ (2002) A case of diffuse leptomeningeal oligodendrogliomatosis associated with HHV-6 variant A. Neuropediatrics 33: 266-270 Doi 10.1055/s-2002-36739

38 Sturm D, Orr BA, Toprak UH, Hovestadt V, Jones DTW, Capper D, Sill M, Buchhalter I, Northcott PA, Leis let al (2016) New Brain Tumor Entities Emerge from Molecular Classification of CNS-PNETs. Cell 164: 1060-1072 Doi 10.1016/j.cell.2016.01.015

39 Tatevossian RG, Lawson AR, Forshew T, Hindley GF, Ellison DW, Sheer D (2010) MAPK pathway activation and the origins of pediatric low-grade astrocytomas. J Cell Physiol 222: 509-514 Doi 10.1002/jcp.21978

40 Thorvaldsdottir H, Robinson JT, Mesirov JP (2013) Integrative Genomics Viewer (IGV): highperformance genomics data visualization and exploration. Brief Bioinform 14: 178-192 Doi 10.1093/bib/bbs017

41 van der Maaten L, Hinton G (2008) Visualizing Data using t-SNE. J Mach Learn Res 9: 2579-2605

42 Zebisch A, Troppmair J (2006) Back to the roots: the remarkable RAF oncogene story. Cell Mol Life Sci 63: 1314-1330 Doi 10.1007/s00018-006-6005-y

43 Zhang J, Wu G, Miller CP, Tatevossian RG, Dalton JD, Tang B, Orisme W, Punchihewa C, Parker M, Qaddoumi let al (2013) Whole-genome sequencing identifies genetic alterations in pediatric lowgrade gliomas. Nat Genet 45: 602-612 Doi 10.1038/ng.2611 
Figure legends.

Figure 1. Molecular classification of DLGNTs by DNA methylation profiling

Unsupervised hierarchical clustering analysis (left panel) of 30 DLGNT samples was performed based on the 10,000 most variably methylated probes. The existence of distinct DLGNT methylation - classes (DLGNT-MC-1, DLGNT-MC2) was demonstrated through unsupervised hierarchical clustering and t-SNE analysis (right panel). DLGNT-MC-1 and DLGNT-MC-2 samples were compared with 129 well-characterized reference samples representing CNS tumors of known histological and/or molecular subtype, confirming the distinct nature of both DLGNT methylation classes.

Figure 2. Radiological features of 3 representative cases of DLGNTs. [DLGNT-MC-1_10 (a-c), DLGNT-MC-2_09 (d-e), DLGNT-MC-2_03 (f)].

Axial and sagittal, T2-weighted MRI images (a-g) reveal T2 hyperintense, multicystic lesions with nodular enhancement in the cerebellum ( $a, b, d)$ and cervical spinal cord spanning several segments (b, c, f) with 'sugar-coating like' leptomeningeal enhancement covering the cerebellum (d, e).

Figure 3. Representative copy-number profiles of DLGNT-MC-1 (a) and DLGNT-MC-2 (b).

Copy-number profiles derived from DNA methylation arrays revealed loss of chromosomal arm $1 \mathrm{p}$ in $100 \%$ of cases. All tumor samples in DLGNT-MC-2 (b) exhibit characteristic gain of chromosomal arm 1q (vs 35\% in DLGNT-MC-1), with several also showing gain of whole chromosome 8 (7/13, 54\% vs $1 / 17,6 \%$ in DLGNT-MC-1). Tumor samples from both subgroups frequently demonstrate a tandem duplication at 7q34 indicating KIAA1549:BRAF fusion (c).

Figure 4. Histopathological features of molecularly defined DLGNT

(a) Case DLGNT-MC-1_09: The DLGNT-MC-1 samples frequently display cells with round, central nuclei and oligodendroglioma-like perinuclear haloes, encompassed by desmoplastic stroma. (b) Case DLGNT-MC-2_10: Tumor cells of the DLGNT-MC-2 entity exhibit small, round nuclei without oligodendroglioma - like perinuclear haloes. Myxoid changes were present in two cases. (c-d) Case DLGNT-MC-1_13: Tumor cells with perinuclear haloes surrounded by neuropil islands were occasionally observed in the DLGNT-MC-1 subgroup. Vascular proliferation was regularly encountered in both subgroups. Mitosis visible (arrow). (e-f) Case DLGNT-MC-1_05: Tumor samples from both subgroups occasionally demonstrated microcysts and hyalinized vessels (e). Rosenthal fibers (f) were frequently encountered, especially in the DLGNT-MC-1 subgroup. Scale bars represent $300 \mu \mathrm{m}$.

Figure 5. Clinical patient information of DLGNT-MC-1 (a) and DLGNT-MC-2 (b).

Tumor location for 13 DLGNT-MC-1 and 12 DLGNT-MC-2 (left panel) are shown. Numbers in circles represent the amount of patients displaying tumor occurrence in the specific region. Patients with multiple tumor sites are counted multiple times. Note: three cases (DLGNT-MC-1_16, DLGNT-MC-1_17 and DLGNT-MC-2_13) were contributed and previously reported by Chiang et al. [9]. Due to preselection based on their exclusive spinal involvement, these cases were excluded in the illustration. Age at diagnosis (middle panel) and gender distribution (right panel) are illustrated with numbers indicating group size.

Figure 6. Molecular DLGNT subgroups correlate with distinct clinical outcome

Kaplan-Meier curves for progression-free (left) and overall (right) survival for molecular DLGNT subgroups defined by methylation profiling (MC-1 and MC-2). 